\title{
Steep decline of HPV 6 and genital warts despite low HPV vaccination coverage in young women in Germany: a long-term, population-based cohort study
}

\section{Agnieszka Denecke}

Klinikum Wolfsburg

Thomas Iftner

University of Tuebingen

Angelika Iftner

University of Tuebingen

Sebastian Riedle

Conreso $\mathrm{GmbH}$

Marion Ocak

MD research

Alexander Luyten

Abts+partner

Isak Üye

Klinikum Wolfsburg

Kübra Tunc

Klinikum Wolfsburg

Karl Ulrich Petry ( $\square$ k.u.petry@klinikum.wolfsburg.de )

Klinikum Wolfsburg

Research article

Keywords: Human papilloma virus, genital warts, condylomata acuminata, HPV-vaccination

Posted Date: October 10th, 2019

DOI: https://doi.org/10.21203/rs.2.15815/v1

License: (1) This work is licensed under a Creative Commons Attribution 4.0 International License.

Read Full License 
Version of Record: A version of this preprint was published at BMC Infectious Diseases on July 2nd, 2021. See the published version at https://doi.org/10.1186/s12879-021-06139-y. 


\section{Abstract}

Background: The introduction of human papilloma virus (HPV) vaccination has resulted in a remarkable decline of genital warts in women and men, but in Germany historical rates of vaccination are relatively low. We report long-term surveillance data on changes in HPV 6 and 11 infection and the prevalence of genital warts in young women in the Wolfsburg HPV epidemiological study (WOLVES).

Methods: Women born in 1983/84, 1988/89, and 1993/94 participated in four cohorts between 2009/10 and 2014/15. Vaccination coverage and prevalence of HPV 6/11 and genital warts are reported for participants aged 19-22 years and 24-27 years at the time of analysis. Statistical analyses were done to compare similarly aged participants using $2 \times 2$ contingency tables (Röhmel-Mansmann unconditional exact test; two-side alpha of 0.05).

Results: A total of 2,456 women were recruited. In 2010, vaccination rates were 40/659 (6.1\%) in women aged $24-27$ years and 142/600 (23.7\%) in those aged 19-22 years; 5 years later, vaccination rates had increased to 135/733 (18.4\%) and 177/368 (48.1\%), respectively. Between 2010 and 2015, there was a statistically significant decrease in the prevalence of HPV 6 among women aged $24-27$ years $(2.12 \%$ versus $0 \% ; P<0.0001)$ and women aged $19-22$ years ( $2.0 \%$ versus $0 \% ; P=0.0056)$. In total, 52 of 2341 participants were diagnosed with genital warts. There was a statistically significant decrease in the lifetime risk of developing genital warts in women aged 24-27 years between 2010 and 2015 (4.7\% versus $1.68 \%$, respectively; $P=0.0018)$. The overall life-time risk of developing genital warts in women aged $19-27$ years decreased from $3.1 \%$ in 2010 to $1.2 \%$ in 2015 ( $P=0.0022)$.

Conclusions: An increase in vaccination coverage was associated with a decreased prevalence of genital warts in young women. A protective effect greater than herd immunity alone was seen despite low vaccination rates. Quadrivalent vaccine had a protective effect on genital HPV 6 positivity and a fully protective effect on the development of genital warts in the youngest population.

\section{Background}

Human papillomavirus (HPV) is one of the most frequently sexually transmitted viral infections in the world (1). Persistent infections with high-risk HPV types (or class I and lla carcinogenic types as defined by the International Agency for Research on Cancer) is a necessary risk factor for the development of cervical cancer, other anogenital malignancies, oropharyngeal cancer, and possibly nonmelanoma skin tumors, while low-risk and other HPV types can lead to benign tumors of the skin and mucosa, for example, genital warts. The term 'genital wart' is not well-defined and covers a wide spectrum of diverse skin and mucosal lesions including the typical condylomata acuminata (2), associated with low-risk HPV genotypes 6 and 11 (3). Risk factors include acquisition of new sex partners, a higher number of sex partners, and concurrent infection with high-risk HPV types (4). Condylomata acuminata are highly contagious and will develop in approximately $65 \%$ of individuals with an infected partner (5). 
Prophylactic HPV vaccines, either the quadrivalent vaccine against HPV types 6, 11 and 16, 18 or the nonavalent vaccine against types $6,11,16,18,31,33,45,52$, and 58 can minimize the overall burden of HPV and genital warts (6). An Australian vaccination program using the quadrivalent HPV vaccine quickly led to a decline and almost complete disappearance of genital warts in both women and men (7). The steep decrease in the prevalence of genital warts occurred before widespread HPV immunization started in boys in 2013 suggesting a herd immunity in the nonvaccinated population of the same age group as the vaccinated girls $(7,8)$.

In contrast to countries with school-based HPV vaccination programs, such as Australia, the UK, and Austria, Germany has a relatively low vaccine coverage. Although HPV vaccination for the prevention of cervical cancer was recommended in March 2007 for females aged 12-17 years, in 2008-2009 the vaccine uptake rate was about $40 \%$ in females aged $16-18$ years and remained at a low $42 \%$ vaccination rate in 2017. Genital warts and HPV infections are not reportable diseases and reliable surveillance data are not available in Germany to estimate the incidence of genital warts and the impact of HPV vaccination (9). Additional data from surveillance studies are necessary to understand the impact of HPV vaccination on the prevalence of HPV infection and associated genital warts. Here we report long-term surveillance data on changes in the prevalence of HPV 6 and 11 and genital warts observed between 2009/10 and 2014/15 in women, aged 19-22 years and 24-27 years, participating in the Wolfsburg HPV epidemiological study (WOLVES).

\section{Methods}

WOLVES is a long-term, population-based cohort study to measure changes in the prevalence of HPV infections and associated diseases after the introduction of HPV vaccines among the general female population from 2009 to 2020 . Women with a first residency in the Wolfsburg area were invited by letter to attend cervical cancer screening in one of 18 private gynecologic practices every year. Voluntary participation in the WOLVES study was open to women irrespective of existing HPV status and all participants gave written informed consent. The study was approved by the local ethics committee (Bo/07/2009).

Participants were recruited in cohorts, defined according to their birth date $(1983 / 84,1988 / 89$, and $1993 / 94)$ and the year of analysis (2009/10 or 2014/15). Four cohorts are reported: cohort $1=$ participants born in 1983/84 and analyzed in 2009/10; cohort 2 = participants born in 1988/89 and analyzed in 2009/10; cohort 3 = participants born in 1988/89 and analyzed in 2014/15; and cohort 4 = participants born in 1993/94 and analyzed in 2014/15. At the time of analysis, participants were aged 19-22 years in cohorts 2 and 4, and aged 24-27 years in cohorts 1 and 3 . Women born in cohort 1 had a single cross-sectional examination in 2009/10, whereas those in cohorts 2 and 3 had annual medical examinations from 2009/10 to 2014/15. Participants in cohort 4 were analyzed in 2014/15 and will have annual visits until the end of 2020 . 
All participants were asked to complete a standardized medical questionnaire that included questions on education, birth country, marital status, pregnancies, parity, contraception, smoking, number of sex partners, age at first intercourse, history of abnormal smears, vaccination status, sexually transmitted infections, and genital warts. During each medical examination, women had a pelvic examination with visualization of the uterine cervix. Pap smears were taken using a spatula and endocervical brush. A second sample was then obtained with a Qiagen Cervical Sampler (Medscan, Uppsala, Sweden) and suspended in $1 \mathrm{ml}$ of specimen transport medium (STM/ Qiagen Inc., Hilden, Germany) for HPV DNA testing. From 2011, samples were taken for liquid cytology using a Cervex brush and ThinPrep (Hologic) transport medium. The gynecologist collected data on HPV vaccination status by checking the certificate of vaccination.

Women were referred to colposcopy if they had genital warts or an abnormal high-grade Pap smear, or they had an abnormal Pap smear classified as borderline or low-grade and tested positive for high-risk HPV. Colposcopy examinations were done at the Klinikum Wolfsburg.

Genital warts were classified as: (i) typical condylomata acuminata for lesions with typical acuminate morphology, which persist on mucosal epithelium or cornifying (cutaneous) skin and have highly vascularized dermal cores producing typical punctuated or cauliflower-like patterns; typical condylomata acuminata tend to be pigmentless and are mostly seen on the pigmented skin (10); (ii) flat condylomata for genital papillomas with a more hyperkeratotic and pigmented surface and flat condylomata with a smooth surface and non-pigmented papules; or (iii) seborrheic wart-like lesions of the cutaneous skin of the external anogenital area. Patients presenting with vulvar intraepithelial neoplasia, Bowenoid papulosis, Naevi or Mollusca contagiosa were excluded (2). Figure 1 shows typical examples of the three types of genital warts observed in the study.

\section{HPV DNA testing}

HPV testing was done using the Hybrid Capture 2 assay (HC2; Qiagen Inc., Hilden Germany). Cervical smear samples were analyzed for the presence of 13 high-risk HPV types $(16,18,31,33,35,39,45,51,52$, $56,58,59$, and 68$)$ and the five low-risk HPV types $(6,11,42,43$, and 44$)$. Samples were diagnosed as positive if they attained or exceeded the FDA-approved threshold of $1.0 \mathrm{pg} \mathrm{HPV} \mathrm{DNA/ml,} \mathrm{which}$ corresponds to 1.0 relative light units (2).

HPV genotyping using SPF-10-PCR, followed by Reverse Line Probe Assay LiPA Extra (SPF-10-PCR) (11) was done on all HC2-positive samples and $10 \%$ of HC2-negative samples. Total DNA was isolated from the cervical samples using a QiaSymphony device (Qiagen, Hilden, Germany) and analyzed by the INNO-LiPA Extra HPV prototype assay (FujiReBioGent, Belgium) according to the manufacturer's instructions. All PCR reactions were done with $10 \mu \mathrm{l}$ input DNA in a final of $50 \mu \mathrm{l}$ using reagents provided by Innogenetics according to the following sequence: $10 \min 37^{\circ} \mathrm{C}, 9 \min 94^{\circ} \mathrm{C}$, and 40 cycles of $30 \mathrm{sec}$ of denaturation at $94^{\circ} \mathrm{C}$, followed by $45 \mathrm{sec}$ at $52^{\circ} \mathrm{C}$ unchanging temperature and $45 \mathrm{sec}$ of extension at $72^{\circ} \mathrm{C}$ run on a MJ Thermocycler PCT 200. The PCR product was then denaturated and a $10 \mu$ aliquot 
hybridized to one strip at $49^{\circ} \mathrm{C}$ for $60 \mathrm{~min}$, followed by multiple washing steps. The INNO-LiPA Extra test allowed identification of 13 established high-risk HPV types $(16,18,31,33,35,39,45,51,52,56,58,59$, and 68), five known or potential high-risk types $(26,53,66,73$, and 82$)$, seven low-risk HPV types (6, 11 , $40,43,44,54$, and 70), additional non-differentiated HPV types, and types with undefined risk $(69,71$, and 74). The strips were analyzed on a flatbed scanner using LiRAS prototype software (Innogenetics, Inc), which displays the patterns and relative intensity of positive bands as arbitrary grey-tone values between 0.1 and 1.0 and allows direct data transfer to Excel spreadsheets. All HC2-low-risk positive samples, which were negative in the LiPA Extra, were retested with the CP4/5 PCR (12). The positive PCR products were purified with the Qiagen Gel Extraction Test QIAquick and then directly sequenced using the CP4 primer.

\section{Study endpoints}

The predefined endpoints were the rates of full HPV vaccination coverage (three doses), the prevalence of HPV 6 and 11, and of genital warts (condylomata acuminata). We reported changes in these prevalence rates according to vaccination status and lifestyle factors (sexual history and smoking history) among participants in the four cohorts.

\section{Statistical analysis}

Vaccination coverage and prevalence rates of HPV 6/11 and genital warts are reported for participants aged 19-22 years (cohorts 2 and 4) and 24-27 years (cohorts 1 and 3) at the time of analysis. Statistical analyses were done to compare similarly aged participants (cohort 1 versus 3 and cohort 2 versus 4) using 2 x 2 contingency tables for the prevalence of HPV 6/11 infection (positive versus negative) and genital warts (no versus yes). The Röhmel-Mansmann unconditional exact test was used to test for difference, with a two-side alpha of 0.05. Statistical analyses were performed using Testimate V.6.5.14.

\section{Results}

Between October 2009 and January 2018, 2,456 women were included. Data are available from 2,360 women for vaccination status and from 2,341 for prevalence of HPV infections and genital warts.

\section{Vaccination coverage}

Table 1 shows vaccination coverage for women in each cohort. During the period 10/2009-12/2010, full vaccine coverage rates were 40/659 (6.1\%) in participants aged 24-27 years (cohort 1) and 142/600 (23.67\%) in those aged 19-22 years (cohort 2). Five years later (11/2014-1/2016), vaccine coverage rates were increased to $135 / 733(18.42 \%)$ and $177 / 368(48.1 \%)$ in participants aged $24-27$ years (cohort 3) and 19-22 years (cohort 4), respectively. 


\section{Prevalence of HPV 6 and 11}

HPV 6 was the most prevalent low-risk HPV type (Table 2). There was a statistically significant decrease in the prevalence of HPV 6 among women aged $24-27$ years $(2.12 \%$ in cohort 1 versus $0 \%$ in cohort 3 ; $P<0.0001$ ) between 2010 and 2015. During this period, there was also a statistically significant decrease in the prevalence of HPV 6 infection among women aged $19-22$ years $(2.0 \%$ in cohort 2 versus $0 \%$ in cohort 4; $\mathrm{P}=0.0056$ ). No cases of HPV 6 positivity were seen in the youngest cohort (cohort 4) during follow-up from 2016 to 2018.

Table 2 shows that the prevalence of HPV 11 was low. Among participants aged 24-27 years, the prevalence of HPV 11 was $0.15 \%$ in cohort 1 and $0.14 \%$ in cohort 3 , whereas in those aged $19-22$ years the rates were $0.67 \%$ in cohort 2 and $0 \%$ in cohort 4 . The observed decline in HPV 11 prevalence was not significant.

\section{Prevalence of genital warts}

Between 2010 and 2015, a total of 52 of 2341 participants were diagnosed with genital warts (Table 3). There was a statistically significant decrease in the life-time risk of developing genital warts between participants in cohort 1 and cohort $3(4.7 \%$ versus $1.68 \%$, respectively; $P=0.0018)$. The difference in the life-time risk of developing genital warts between cohorts 2 and $4(1.33 \%$ versus $0.27 \%)$ was not statistically significant and there was only one case reported in cohort 4 . There was a statistically significant decrease in the overall life-time risk of developing genital warts between participants analyzed in 2010 (cohorts 1 and 2) and those in 2015 (cohorts 3 and 4): $3.1 \%$ versus $1.2 \%$, respectively; $P=0.0022$.

The numbers of incident genital warts diagnosed in the colposcopy clinic in the periods 2010-11 and 2015-16 were as follows: five cases in cohort 1, four cases in cohort 2, three cases in cohort 3 and no case in cohort 4. Out of the nine cases diagnosed in 2010-11, five were classified as typical condylomata acuminata, two as flat condylomata and two as seborrheic wart-like. The three cases diagnosed in 2015-16 were classified as typical condylomata acuminata (one case) and seborrheic wart-like (two cases). All cases with incident typical condylomata acuminata were associated with detection of HPV 6 in cervical samples and in typical condylomata acuminata-tissue.

The cervical smear samples of 5 of 45 patients $(11.1 \%)$ with genital warts tested positive for HPV 6 or 11 , while 17 of 45 (37.78\%) tested positive for HPV 6, 11, and high-risk types. The presence of HPV 6 in cervical samples directly correlated to the genesis and development of genital warts, while co-infection with HPV 6 and 11 was found in only one case.

Table 4 shows the prevalence of genital warts in the cohort with the lowest vaccine coverage $(6.1 \%$ in cohort 1) compared with cohorts with greater coverage. An increase in the rate of vaccine coverage was associated with a decrease in the prevalence of genital warts. 


\section{Lifestyle factors}

Complete sexual behavior and smoking history data in this large population of women showed some differences between cohorts. The mean age at first intercourse was 16.9 years in cohort 1, 16.5 years in cohorts 2 and 3 , and 16.2 in cohort 4 but this decline was not significant. There were no remarkable differences in numbers of sexual partners

Results of univariate testing (Wilcoxon-Mann-Withney-U-Test) and multivariate testing (logistic regression) showed a significant correlation between the number of sexual partners and risk of genital warts in all cohorts $(p<0.0001)$. Young age at first intercourse and currently smoking were not associated with an increased risk for genital warts. We observed a decline in the proportion of current smokers between 2010 and 2015. In 2010, 36.8\% of cohort 2 were smokers compared with $28.2 \%$ in cohort 4 five years later. The corresponding rates for current smokers were $37.0 \%$ in cohort 1 in 2010 and $27.4 \%$ in cohort 3 in 2015.

\section{Discussion}

WOLVES is the first prospective, real-life population-based epidemiological study to measure the impact of HPV vaccination on the prevalence of low-risk HPV and genital warts in Germany only a few years after the introduction of HPV vaccination. In this analysis, we observed a steep decline in the life-time risk of genital warts in women in their mid-twenties from 4.7\% among women born in 1983/4 and analyzed in 2010 to $1.7 \%$ in those born in $1988 / 9$ and analyzed in 2015 . The decrease in genital warts was accompanied by a significant reduction in the prevalence of HPV 6 infection, however, the very low prevalence of HPV 11 had no obvious impact on the development of genital warts. Somewhat unexpectedly, the decrease in genital warts and HPV 6 infection was observed despite the low vaccination coverage in our population $(<50 \%)$. It is possible that a single HPV dose may be protective, as suggested by recent publications $(13,14)$. In this study, we did not observe a single case of persistent infection with HPV 6, 11, 16, or 18 in women who received one dose of vaccine. Inclusion of all women who received at least 1 dose of vaccine would have resulted in a vaccine coverage rate of $52 \%$ in cohort 4 in this analysis.

Previous studies have shown that high levels of HPV vaccine coverage may virtually eliminate genital warts in young women and men (reviewed in $(15,16)$ ). Australia and other countries with a high vaccine coverage already observed the near-extinction of genital warts in adolescents and young adults (17). Surveillance studies from countries with vaccination programs based on bivalent HPV vaccine and from countries with low vaccine coverage showed a less pronounced decline in the incidence of genital warts. Recent publications showed the early benefit of vaccination such as the decrease in the incidence of anogenital warts in women aged $<22$ years and the possible development of herd immunity within population with high vaccination coverage (18).

The results from our study also show a clear correlation between an increase in vaccination coverage and a reduction in the life-time risk of developing genital warts. In WOLVES, it was notable that a decline of 
genital warts and complete disappearance of HPV 6 was observed in young women with the highest vaccination coverage, i.e. those aged 19-22 years. Nevertheless, the vaccination coverage in our study population was lower than in previously conducted studies, but seems to be high enough to confer herd immunity (19). A systematic review and meta-analysis of transmission-dynamic models concluded that strong herd effects are expected from vaccinating girls only, even with coverage rates as low as $20 \%$ (20). Other studies in Germany have also suggested the possibility of herd immunity despite low vaccine coverage. Mikolajczyk et al. reported a decrease of the incidence of genital warts in females aged 16 years (47\%), 17 years (45\%) and 18 years (35\%) at the end of 2008 , one year after the recommended introduction of HPV vaccination (21). Furthermore, Thöne et al. reported a maximum reduction of up to $60 \%$ in genital warts among 16 - to 20 -year-old females and no decrease for corresponding groups outside the range 14-24 years (22). We interpret our data as a proof that transmission of HPV 6 as the causal agent of condylomata acuminata and even of other genital warts is strongly inhibited even in populations with low vaccination coverage, although the exact mechanism of this protection is unknown and the necessary level of vaccination coverage remains uncertain (23).

The major limitation of this analysis is the low number of genital warts reported in our population (52 cases). An increase in the use of self-applied topical treatment of genital warts over time may have contributed to the decline in the surgical treatments seen in our analysis, however, such an effect would be expected in all cohorts and, therefore, the decline observed in our youngest cohort (1993/94) cannot be explained by changes in the topical treatment of genital warts alone. It is possible that some selfreported diagnoses of genital warts were not valid and the true prevalence could be higher than reported in our previous analysis (2). The rate of underdiagnosed women is probably not related to vaccination status and the low occurrence of genital warts did not have any impact on our analyses.

The major strengths of this study are the recruitment of a large general population and the prospective nature of the design, rather than being only a retrospective analysis of existing databases. We recruited women aged 19 to 27 years and to our knowledge, this is the first complete population-based analysis of low-risk HPV and genital wart prevalence data from women in this age group in Germany. Between 2015 and 2019, final analyses of all colposcopies and subsequent procedures were done to ensure that the clinical data were robust. The analysis is also strengthened by collection of comprehensive behavioral and lifestyle data, which show that the observed reduction of genital warts is unlikely to be explained by changes in sexual behavior and smoking.

\section{Conclusions}

This analysis of the ongoing WOLVES study adds evidence about the short-term impact of HPV vaccination and shows protection against HPV 6-related genital warts despite low vaccine coverage. Furthermore, an increase in the vaccination coverage rate was associated with decreased prevalence of genital warts in young women. We observed a protective effect with the quadrivalent vaccine on genital HPV 6 positivity and a fully protective effect on the development of genital warts in the youngest population. We believe that further monitoring of this trend is very important to evaluate the progress of 
state-sponsored HPV vaccination policies in Germany. Although previous studies and meta-analysis demonstrated the effectiveness of HPV vaccination programs, less is known about the population effectiveness of HPV vaccination and its short-term effects on HPV-related health disparities.

\section{List Of Abbreviations}

HC2 = Hybrid Capture 2; HPV = human papilloma virus; $\mathrm{PCR}=$ polymerase chain reaction; WOLVES = Wolfsburg HPV epidemiological study

\section{Declarations}

Ethics approval and consent to participate: The ethics committee of Lower Saxony in Hannover approved WOLVES as an epidemiological non-interventional study ( $\mathrm{Nr} \mathrm{Bo/07/2009).} \mathrm{All} \mathrm{participants} \mathrm{gave} \mathrm{written}$ informed consent.

Consent for publication: Not applicable.

Availability of data and material: WOLVES is managed by Klinikum Wolfsburg, the central database is located in the department of gynecology. The contract between the financier, gynecologists in private practice, other partners, and Klinikum Wolfsburg encourages the use of WOLVES data for research and gives all rights for analyses and publications to the head of the department of obstetrics and gynecology as leader of the scientific team.

Competing interests: KUP received an honorarium from Merck-MSD for participation in an advisory board. TI has patents for "Primer and probes for detection of papillomaviruses" DE 10357677 A1 (Co-Inventor, T. Iftner; ownership, Greiner Bio-One $\mathrm{GmbH}$ ) and "Detection of human papillomaviruses" European patent and EP Swiss EPÜ 1272675 (Inventor, T. Iftner; ownership, Eberhard-Karls-Universitaet Tuebingen, Germany), has received speaker honoraria from Hologic, BD Diagnostics, and MSD, and has received unconditional research grants to the University Hospital of Tuebingen from Hologic, USA (IIT) and BectonDickinson, Germany (IIT). The other authors declare that they have no competing interests.

Funding: WOLVES is an epidemiological study of Klinikum Wolfsburg. The study was financed by MerckMSD with an unrestricted grant. The financier had no role in the study design, study conduct, data analysis, or reporting of results. Hologic supported WOLVES with ThinPrep vials free of charge.

Authors' contributions: AD drafted the manuscript, collected data, and examined patients. KUP is the scientific leader of WOLVES and responsible for the overall study protocol and the concept of the submitted analysis, MO did the statistical analyses for this publication, Al and TI were responsible for HPV testing, AL collected data and examined patients, KT was responsible for data documentation. All authors have reviewed and approved the final version of the manuscript and consent to publication. 
Acknowledgements: We would like to thank all participating gynaecologists, all WOLVES participants and staff members of Klinikum Wolfsburg and associated facilities for their support. The manuscript was corrected for language by Tim Kelly, Medi-Kelsey, who was paid by the authors.

\section{References}

1.Patel $H$, Wagner M, Singhal P, Kothari S. Systematic review of the incidence and prevalence of genital warts. BMC Infect Dis. 2013;13:39.

2.Petry KU, Luyten A, Justus A, Iftner A, Strehlke S, Schulze-Rath R, Iftner T. Prevalence of low-risk HPV types and genital warts in women born 1988/89 or 1983/84 -results of WOLVES, a population-based epidemiological study in Wolfsburg, Germany. BMC Infect Dis. 2012;12:367.

3.Gross G, Hagedorn M, Ikenberg H, Rufli T, Dahlet C, Grosshans E, Gissmann L. Bowenoid papulosis. Presence of human papillomavirus (HPV) structural antigens and of HPV 16-related DNA sequences. Arch Dermatol. 1985;121:858-63.

4.Garland SM, Steben M, Sings HL, James M, Lu S, Railkar R, et al. Natural history of genital warts: analysis of the placebo arm of 2 randomized phase III trials of a quadrivalent human papillomavirus (types 6, 11, 16, and 18) vaccine. J Infect Dis. 2009;199:805-14.

5.Lacey CJ, Lowndes CM, Shah KV. Chapter 4: Burden and management of non-cancerous HPV-related conditions: HPV-6/11 disease. Vaccine. 2006;24 Suppl 3:S3/35-41.

6.Navarro-Illana E, Lopez-Lacort M, Navarro-Illana P, Vilata JJ, Diez-Domingo J. Effectiveness of HPV vaccines against genital warts in women from Valencia, Spain. Vaccine. 2017;35:3342-6.

7.Ali H, McManus H, O'Connor CC, Callander D, Kong M, Graham S, et al. Human papillomavirus vaccination and genital warts in young Indigenous Australians: national sentinel surveillance data. Med $\mathrm{J}$ Aust. 2017;206:204-9.

8.Tabrizi SN, Brotherton JM, Kaldor JM, Skinner SR, Liu B, Bateson D, et al. Assessment of herd immunity and cross-protection after a human papillomavirus vaccination programme in Australia: a repeat crosssectional study. Lancet Infect Dis. 2014;14:958-66.

9. Hillemanns P, Breugelmans JG, Gieseking F, Benard S, Lamure E, Littlewood KJ, Petry KU. Estimation of the incidence of genital warts and the cost of illness in Germany: a cross-sectional study. BMC Infect Dis. 2008;8:76.

10.von Krogh G, Lacey CJ, Gross G, Barrasso R, Schneider A. European course on HPV associated pathology: guidelines for primary care physicians for the diagnosis and management of anogenital warts. Sex Transm Infect. 2000;76:162-8. 
11.Klug SJ, Molijn A, Schopp B, Holz B, Iftner A, Quint W, et al. Comparison of the performance of different HPV genotyping methods for detecting genital HPV types. J Med Virol. 2008;80:1264-74.

12.Iftner A, Klug SJ, Garbe C, Blum A, Stancu A, Wilczynski SP, Iftner T. The prevalence of human papillomavirus genotypes in nonmelanoma skin cancers of nonimmunosuppressed individuals identifies high-risk genital types as possible risk factors. Cancer Res. 2003;63:7515-9.

13.Dehlendorff C, Sparen P, Baldur-Felskov B, Herweijer E, Arnheim-Dahlstrom L, Ploner A, et al. Effectiveness of varying number of doses and timing between doses of quadrivalent HPV vaccine against severe cervical lesions. Vaccine. 2018;36:6373-8.

14. Kreimer AR, Herrero R, Sampson JN, Porras C, Lowy DR, Schiller JT, et al Evidence for single-dose protection by the bivalent HPV vaccine-Review of the Costa Rica HPV vaccine trial and future research studies. Vaccine. 2018;36:4774-82.

15.Garland SM, Kjaer SK, Munoz N, Block SL, Brown DR, DiNubile MJ, et al. Impact and effectiveness of the quadrivalent human papillomavirus vaccine: a systematic review of 10 years of real-world experience. Clin Infect Dis. 2016;63:519-27.

16.Drolet M, Benard E, Perez N, Brisson M, Group HPVVIS. Population-level impact and herd effects following the introduction of human papillomavirus vaccination programmes: updated systematic review and meta-analysis. Lancet. 2019;394:497-509.

17.Patel C, Brotherton JM, Pillsbury A, Jayasinghe S, Donovan B, Macartney K, Marshall H. The impact of 10 years of human papillomavirus (HPV) vaccination in Australia: what additional disease burden will a nonavalent vaccine prevent? Euro Surveill. 2018; doi: 10.2807/1560-7917.ES.2018.23.41.170073.

18.Smith LM, Strumpf EC, Kaufman JS, Lofters A, Schwandt M, Levesque LE. The early benefits of human papillomavirus vaccination on cervical dysplasia and anogenital warts. Pediatrics. 2015;135:e1131-40.

19.Ali H, Guy RJ, Wand H, Read TR, Regan DG, Grulich AE, et al. Decline in in-patient treatments of genital warts among young Australians following the national HPV vaccination program. BMC Infect Dis. 2013;13:140.

20.Brisson M, Benard E, Drolet M, Bogaards JA, Baussano I, Vanska S, et al. Population-level impact, herd immunity, and elimination after human papillomavirus vaccination: a systematic review and metaanalysis of predictions from transmission-dynamic models. Lancet Public Health. 2016;1:e8-e17.

21.Mikolajczyk RT, Kraut AA, Horn J, Schulze-Rath R, Garbe E. Changes in incidence of anogenital warts diagnoses after the introduction of human papillomavirus vaccination in Germany-an ecologic study. Sex Transm Dis. 2013;40:28-31. 
22.Thone K, Horn J, Mikolajczyk R. Evaluation of vaccination herd immunity effects for anogenital warts in a low coverage setting with human papillomavirus vaccine-an interrupted time series analysis from 2005 to 2010 using health insurance data. BMC Infect Dis. 2017;17:564.

23.Kahn JA, Brown DR, Ding L, Widdice LE, Shew ML, Glynn S, Bernstein DI. Vaccine-type human papillomavirus and evidence of herd protection after vaccine introduction. Pediatrics. 2012;130:e249-56.

\section{Tables}

Table 1. HPV vaccination status among the WOLVES cohorts. Full vaccination status is defined as 3 doses.

\begin{tabular}{|c|c|c|c|c|}
\hline \multirow[t]{2}{*}{ Cohort* } & \multirow[t]{2}{*}{ Year of analysis } & \multirow[t]{2}{*}{ Age at time of analysis (y) } & \multicolumn{2}{|c|}{ Full vaccination status, $\mathbf{N}(\%)$} \\
\hline & & & No & Yes \\
\hline \multirow[t]{2}{*}{1} & $2009 / 10$ & $24-27$ & $619^{a}$ & 40 \\
\hline & & & (93.93\%) & (6.1\%) \\
\hline \multirow[t]{2}{*}{3} & $2014 / 15$ & & $598^{\mathrm{b}}$ & 135 \\
\hline & & & (81.58\%) & (18.42\%) \\
\hline \multirow[t]{2}{*}{2} & $2009 / 10$ & $19-22$ & $458^{\mathrm{c}}$ & 142 \\
\hline & & & $(76.33 \%)$ & $(23.67 \%)$ \\
\hline \multirow[t]{2}{*}{4} & $2014 / 15$ & & $191^{\mathrm{d}}$ & 177 \\
\hline & & & (51.9\%) & (48.09\%) \\
\hline
\end{tabular}

*Cohort 1 born 1983/84 analyzed in 2009/10; cohort 2 born in 1988/89 analyzed in 2009/10; cohort 3 born 1988/89 analyzed in 2014/15; cohort 4 born 1993/94 analyzed in 2014/15

a 2 women received 1 dose; ${ }^{b} 4$ women received 1 dose and 7 received 2 doses; ${ }^{c} 3$ women received 1 dose and 9 received 2 doses; ${ }^{d} 5$ women received 1 dose and 8 received 2 doses

Table 2. Prevalence of HPV 6 and HPV 11 among WOLVES cohorts (2010-2015). Statistical comparisons are shown for cohorts 1 versus 3 and cohorts 2 versus 4 . 


\begin{tabular}{lccccccc}
\hline Cohort* & N & \multicolumn{2}{c}{ HPV 6 } & Rate difference & \multicolumn{2}{c}{ HPV 11 } & Rate difference \\
& & Negative & Positive & $(95 \%$ CI) & Negative & Positive & (95\% CI \\
\hline 1 & 659 & 645 & 14 & -0.0212 & 658 & 1 & -0.0001 \\
& & $(97.88 \%)$ & $(2.12 \%)$ & $(-0.039,-0.01)$ & $(99.85 \%)$ & $(0.15 \%)$ & $(-0.0083,0.0075)$ \\
3 & 714 & 714 & 0 & P<0.0001 & 713 & 1 & $\mathrm{P}=1.0000$ \\
& & $(100 \%)$ & $(0 \%)$ & & $(99.86 \%)$ & $(0.14 \%)$ & \\
\hline 2 & 600 & 588 & 12 & -0.02 & 596 & 4 & -0.0067 \\
& & $(98 \%)$ & $(2 \%)$ & $(-0.0383,-0.0063)$ & $(99.33 \%)$ & $(0.67 \%)$ & $(-0.0197,0.0052)$ \\
4 & 368 & 368 & 0 & $P=0.0056$ & 368 & 0 & $P=0.2753$ \\
& & $(100 \%)$ & $(0 \%)$ & & $(100 \%)$ & $(0 \%)$ & \\
\hline
\end{tabular}

*Cohort 1 born 1983/84 analyzed in 2009/10; cohort 2 born in 1988/89 analyzed in 2009/10; cohort 3 born 1988/89 analyzed in 2014/15; cohort 4 born 1993/94 analyzed in 2014/15

Table 3. Life-time risk (sum of pre-existing and incident cases) of developing genital warts in WOLVES cohorts (2010-2015). Statistical comparisons are shown for cohorts 1 versus 3, cohorts 2 versus 4, and participants analyzed in 2010 (cohorts $1+2$ ) versus those analyzed in 2015 (cohorts $3+4$ ). 


\begin{tabular}{|c|c|c|c|c|}
\hline \multirow[t]{2}{*}{ Cohort* } & \multirow[t]{2}{*}{$\mathbf{N}$} & \multicolumn{2}{|c|}{ Genital warts } & \multirow{2}{*}{$\begin{array}{l}\text { Rate difference } \\
\qquad(95 \% \mathrm{CI})\end{array}$} \\
\hline & & No & Yes & \\
\hline \multirow[t]{2}{*}{1} & 659 & 628 & 31 & 0.0302 \\
\hline & & (95.29\%) & $(4.7 \%)$ & $(0.0109,0.052)$ \\
\hline \multirow[t]{2}{*}{3} & 714 & 702 & 12 & $\mathrm{P}=0.0018$ \\
\hline & & $(98.32 \%)$ & $(1.68 \%)$ & \\
\hline \multirow[t]{2}{*}{2} & 600 & 592 & 8 & 0.0106 \\
\hline & & $(98.67 \%)$ & $(1.33 \%)$ & $(-0.0042,0.0255)$ \\
\hline \multirow[t]{2}{*}{4} & 368 & 367 & 1 & $P=0.1598$ \\
\hline & & $(99.72 \%)$ & $(0.27 \%)$ & \\
\hline \multirow[t]{2}{*}{$1+2$} & 1259 & 1220 & 39 & 0.019 \\
\hline & & (96.9\%) & (3.1\%) & $(0.0068,0.032)$ \\
\hline \multirow[t]{2}{*}{$3+4$} & 1082 & 1069 & 13 & $\mathrm{P}=0.0022$ \\
\hline & & $(98.8 \%)$ & $(1.2 \%)$ & \\
\hline
\end{tabular}

*Cohort 1 born 1983/84 analyzed in 2009/10; cohort 2 born in 1988/89 analyzed in 2009/10; cohort 3 born 1988/89 analyzed in 2014/15; cohort 4 born 1993/94 analyzed in 2014/15

Table 4. Prevalence of genital warts according to vaccination status in the WOLVES cohorts.

\begin{tabular}{lcccccc}
\hline & \multicolumn{3}{c}{ Cohort } & \multicolumn{3}{c}{ Difference between cohorts } \\
\cline { 2 - 7 } & 1 & 3 & 4 & 1 to 3 & 3 to 4 & 1 to 4 \\
\hline $\mathrm{N}$ & 659 & 733 & 368 & -74 & 365 & 291 \\
Vaccinated & 40 & 135 & 177 & -95 & -42 & -137 \\
\hline Unvaccinated & 619 & 598 & 191 & 21 & 407 & 428 \\
GW & 26 & 18 & 1 & -8 & -17 & -25 \\
$(\%$ change) & & & & $(-30.77 \%)$ & $(-65.38 \%)$ & $(-96.15 \%)$ \\
\hline GW/Vaccinated & $1 / 40$ & $3 / 135$ & $1 / 191$ & +2 & -2 & 0 \\
(\% change) & & & & $(+1.1 \%)$ & $(-61 \%)$ & $(-100 \%)$ \\
\hline
\end{tabular}




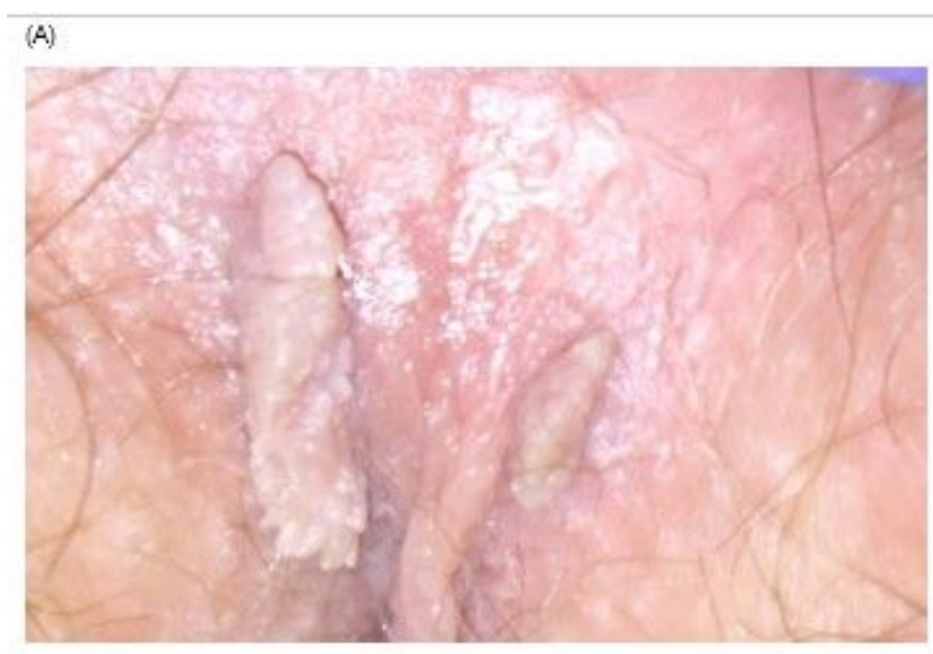

(B)

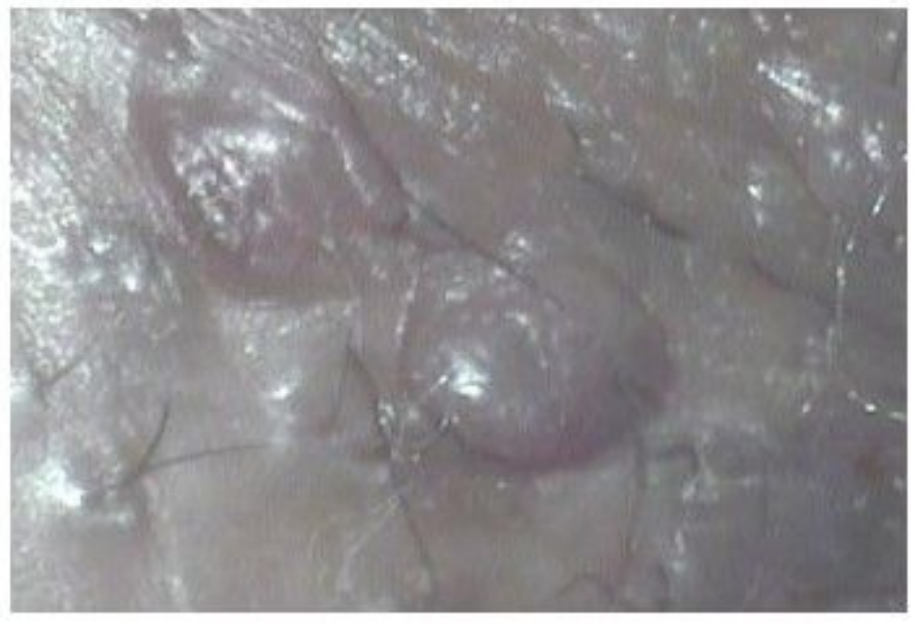

(C)

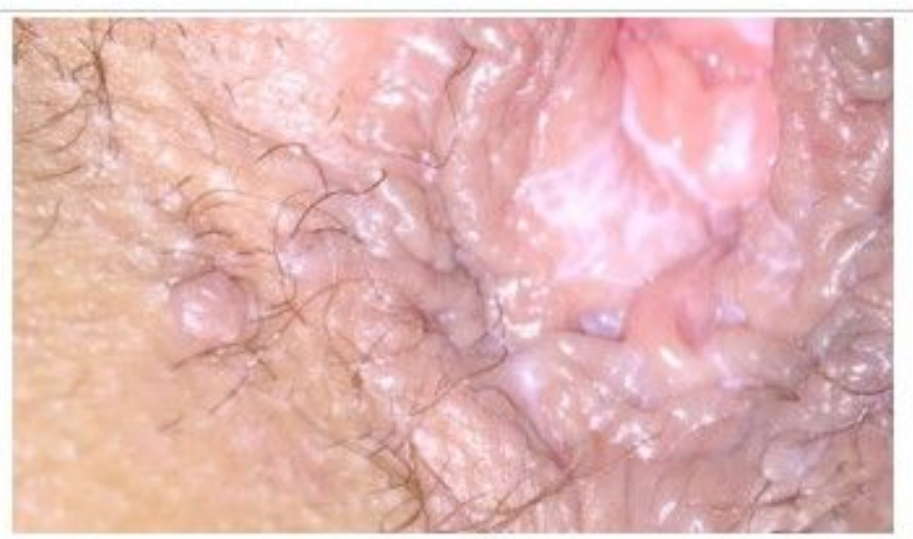

Figure 1

Examples of (A) typical condylomata acuminata, (B) flat condylomata, and (C) seborrheic wart-like lesions. 